\title{
A Sex-Limited Serum Protein Variant in the Mouse: Hormonal Control of Phenotypic Expression
}

\author{
Howard C. Passmore ${ }^{1}$ and Donald C. Shreffler ${ }^{1}$
}

Received 2 Oct. 1970-Final 17 Nov. 1970

The sex-limited protein (Slp) antigen of the mouse is first detected in the serum of strain $D B A / 2 J$ males at 5- 6 weeks of age and reaches full adult levels by 10 weeks. This antigen is normally absent in females. Immature $D B A / 2 J$ males castrated at $3 \frac{1}{2}$ weeks of age failed to develop Slp antigen, while DBA/2J females treated with testosterone propionate starting at $3 \frac{1}{2}$ weeks developed normal adult male levels of Slp antigen. Similar hormoneinfluenced effects were demonstrated in adult males and females of the same strain. Experiments indicated that testosterone does not act directly in the serum to expose Slp antigenic sites. Testosterone treatment of both males and females of strain C57BL/ 10JSf, which does not carry the gene for the presence of the Slp antigen, failed to stimulate the appearance of the antigen. Thus, the presence of Slp antigen in the serum is dependent on both the proper genotype and the presence of male hormone.

\section{INTRODUCTION}

Genetically controlled biochemical variants in mammals may be of particular interest and importance where they can be used as tools for exploring interactions between specific gene loci and the physiology of the animal. The sex-limited protein (Slp) variant of the mouse (Passmore and Shreffler, 1970) provides an unusual opportunity for examining the interaction of genetic and hormonal control on the phenotypic expression of a serum protein antigen. The sex-limited serum protein variant was discovered during a search for structural differences in the Ss component of mouse serum, which exhibits genetically controlled quantitative variation (Shreffler and Owen, 1963; Shreffler, 1967).

The basic characteristics of the Slp variant system are: (1) The Slp antigen is detected by a specific alloantiserum on agar immunodiffusion. (2) The presence of

\footnotetext{
Supported by U.S.P.H.S. Research Grant GM-15419, U.S.P.H.S. Training Grant 2T01-GM-00071 (H.C.P.), and U.S.P.H.S. Career Development Award K3-HE-24,980 (D.C.S.).

1 Department of Human Genetics, University of Michigan, Ann Arbor, Michigan.
} 
the antigen in the serum is determined by two alleles at an autosomal locus, with the presence of the Slp antigen being determined by a dominant allele $\left(S l p^{a}\right)$. The Slp antigen is not detectable in the serum of the recessive homozygote $\left(S l p^{o} / S l p^{o}\right)$. (3) The Slp locus maps within the histocompatibility-2 region in linkage group IX, and in the same map position as the genetic determinant for quantitative variation in the Ss protein. (4) Immunological evidence indicates that the Slp variant reflects structural variation or modification in the Ss protein. (5) The Slp antigen is normally found only in mature males of genotypically positive strains, such as $\mathrm{DBA} / 2 \mathrm{~J}$ and $\mathrm{A} / \mathrm{J}$. It is absent from males of negative strains (C57BL/10JSf, C3H/JSf, and $\mathrm{RF} / \mathrm{J}$ ) and is phenotypically absent from females of all strains. This trait is therefore sex-limited; i.e., expression is limited to the male sex. A strain of mice is designated Slp-positive if serum from mature males of that strain types positive for the Slp antigen or designated Slp-negative if serum from mature males lacks the antigen. The investigations described in this report were designed to examine the basis for this sex-limitation, and more specifically to determine the effect of male hormone on expression of the Slp antigen. A preliminary report of this work has been published (Passmore, 1969).

\section{METHODS}

Inbred strains of mice were obtained from either the Jackson Laboratory, Bar Harbor, Maine (DBA/2J and RF/J), or from our own colony at the University of Michigan (C57BL/10JSf, C3H/JSf, and A/Sn).

All alloimmunizations were carried out according to the procedures of Passmore

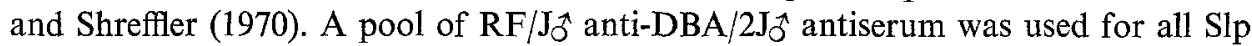
typing and quantitation. Rabbit anti-Ss was produced according to the procedure of Shreffler and Owen (1963).

DBA/2J males were castrated at $3 \frac{1}{2}$ or 12 weeks of age by making a dorsal incision into the peritoneal cavity after anesthetizing with sodium pentabarbital. The testes were pulled from the body cavity, ligated at the ductuli efferentes, and excised. The sham control consisted of anesthesia, incision, pulling the testes out of the body cavity, mild probing, and then replacing the testes in the peritoneal cavity.

Testosterone propionate (Calbiochem) was injected subcutaneously using sesame oil as a carrier. The dosages used were as follows: (1) Animals treated from $3 \frac{1}{2}$ to 10 weeks of age received $1.5 \mathrm{mg}$ of testosterone propionate per week in two injections for the first 10 days and then $2.25 \mathrm{mg}$ of hormone per week in three injections for the remainder of the treatment period. (2) Animals in which treatment was started at 1 week of age received gradually increasing doses of testosterone propionate equivalent to $0.1 \mathrm{mg} /$ week/gram of body weight in two or three injections per week. This dose is equivalent to the adult dose of $2.25 \mathrm{mg}$ per week. (3) Females in which treatment was started at 12 weeks of age received $2.25 \mathrm{mg}$ of the hormone per week in three injections. (4) An eightfold increased dose of testosterone propionate was used in one experiment, consisting of $18.0 \mathrm{mg}$ of hormone per week.

Immunodiffusion was carried out according to the method described by Passmore and Shreffler (1970), with the exception that 8 by $10 \mathrm{~cm}$ glass plates, each with $12 \mathrm{ml}$ of 
agar gel, were used instead of 2.5 by $7.5 \mathrm{~cm}$ microscope slides. This allowed for more typing wells per plate and provided a flatter gel surface.

The quantity of Slp antigen in individual serum samples was estimated on immunodiffusion plates by measuring the distance from the antigen well to the precipitin line and expressing this distance as a percentage of the total distance between the antigen and antibody wells, then comparing these $R_{f}$ values with values obtained by standard dilutions of the Slp antigen. Since a standard pool of antiserum (RF/J $\mathbf{J}_{\bigcirc}$ anti-DBA $\left./ 2 \mathbf{J}_{\circlearrowleft}\right)$ was used in all tests, precipitin band position was solely a function of antigen concentration. The level of the Slp antigen in the castration and hormone treatment studies was estimated in this way using a pool of adult DBA/2J male serum (4-8 months of age) as the $100 \%$ standard. Twenty microliters of antigen or antibody was used in all wells. This technique reliably distinguished between twofold serial dilutions, i.e., between undiluted antigen and a one-half dilution, between one-half and one-fourth dilutions, etc.

The Ss protein was quantitated by double immunodiffusion in tubes, according to the method of Preer (1956) as modified by Shreffler and Steinberg (1967).

\section{RESULTS}

The Slp antigen is first detected in trace amounts in normal DBA/2J male serum at 5-6 weeks of age, a time which approximately corresponds to the onset of sexual maturity. The level of antigen then begins to increase until the adult level is reached at about 10 weeks of age. However, immature DBA/2J males which were castrated at $3 \frac{1}{2}$ weeks of age failed to develop Slp antigen and were still negative for the antigen when tested after 25 weeks of age (Fig. 1). When these castrated males were then treated with testosterone propionate beginning at 25 weeks, Slp antigen was detected 7 days after the start of treatment. In sham-operated DBA/2J males, the Slp development was normal. Animals which were castrated, but then received replacement therapy with testosterone propionate, developed normal levels of Slp at a rate very similar to that of untreated males.

To further test the effect of male hormone on expression of the Slp variant, females were treated with testosterone propionate from $3 \frac{1}{2}$ to 10 weeks of age. As illustrated in Fig. 2, these animals developed normal male levels of Slp by 10 weeks of age. Females that were first ovariectomized and then treated with testosterone showed a similar appearance of Slp. The slope and shape of these two curves are almost identical to those of untreated males in Fig. 1. Control animals which were ovariectomized and treated with sesame oil, the carrier for the testosterone propionate, did not develop Slp antigen.

In the preceding experiments, in which treatment of females or castrated males was started at $3 \frac{1}{2}$ weeks of age, the Slp antigen began to appear at about 5 weeks of age. It was of interest to know whether it was possible to make the Slp antigen appear earlier in postnatal development by beginning the hormone treatment earlier. In an experiment in which hormone treatment was started at 1 week rather than $3 \frac{1}{2}$ weeks of age, in two litters containing both males and females, the Slp antigen appeared again at 5 weeks of age, i.e., no earlier than in the previous experiment 


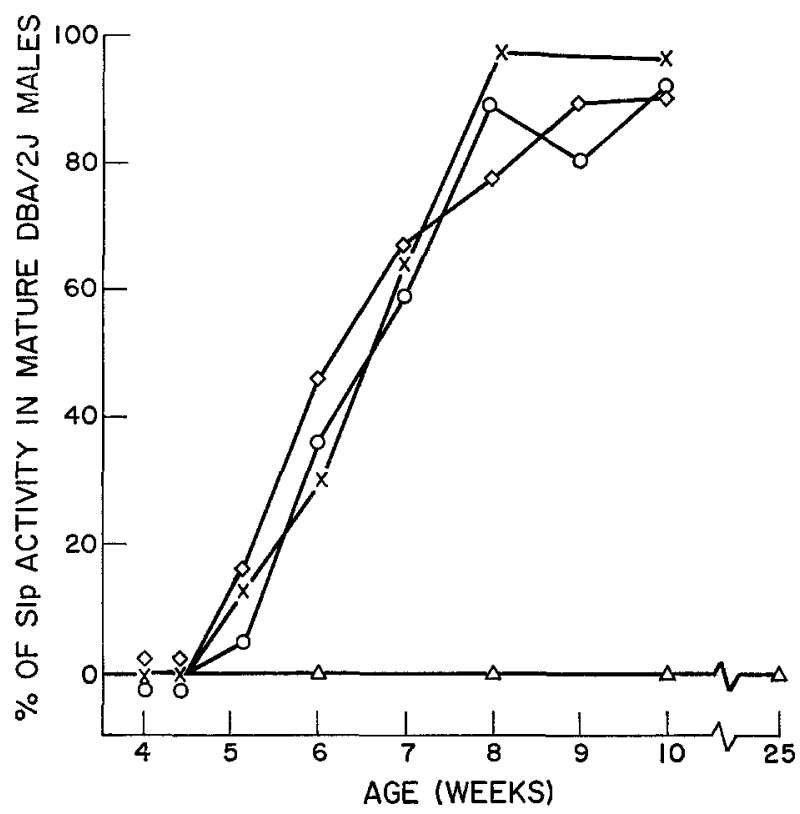

Fig. 1. The effect of castration on immature $\mathrm{DBA} / 2 \mathrm{~J}$ males: -O- sham control males (four animals); $-\Delta-$ castrated (eight animals); $\diamond-$ - castrated + testosterone beginning at $3 \frac{1}{2}$ weeks of age (four animals); $-\times-$ males + testosterone beginning at 1 week of age (four animals).

(see Figs. 1 and 2). These animals developed the antigen at the same rate as animals started on testosterone treatment at $3 \frac{1}{2}$ weeks of age.

The failure of castrated males to develop Slp and the ability of females treated with testosterone propionate to develop the antigen strongly suggest that male hormone triggers the appearance of Slp antigen in genotypically positive strains, such as DBA/ $2 \mathrm{~J}$. Treatment of both males and females of a genetically negative strain such as C57/ $\mathrm{BL} / 10 \mathrm{JSf}$ with testosterone propionate failed to induce the appearance of S1p antigen.

Similar studies of the effects of castration and hormone treatment on Slp activity were carried out with mature 12-week-old animals. At 12 weeks of age, DBA/2J males have already developed full levels of Slp antigen. Males castrated at this age showed a sharp reduction in quantitative level of Slp to about $30 \%$ of the normal adult level by 21 days after castration (Fig. 3). After this rapid drop, the level of antigen decreased at a much slower rate, reaching $8-10 \%$ by 150 days after castration, and by 200 days falling to a level below the smallest quantity detectable by the immunodiffusion technique (less than $5 \%$ ). Untreated sham controls maintained $100 \% \mathrm{Slp}$ levels throughout the experiment. Castration of DBA/2J adult males, therefore, results in the loss of all detectable amounts of Slp antigen. When two of these castrated males were treated with testosterone propionate after 60 days, both showed a return to full adult male levels. 


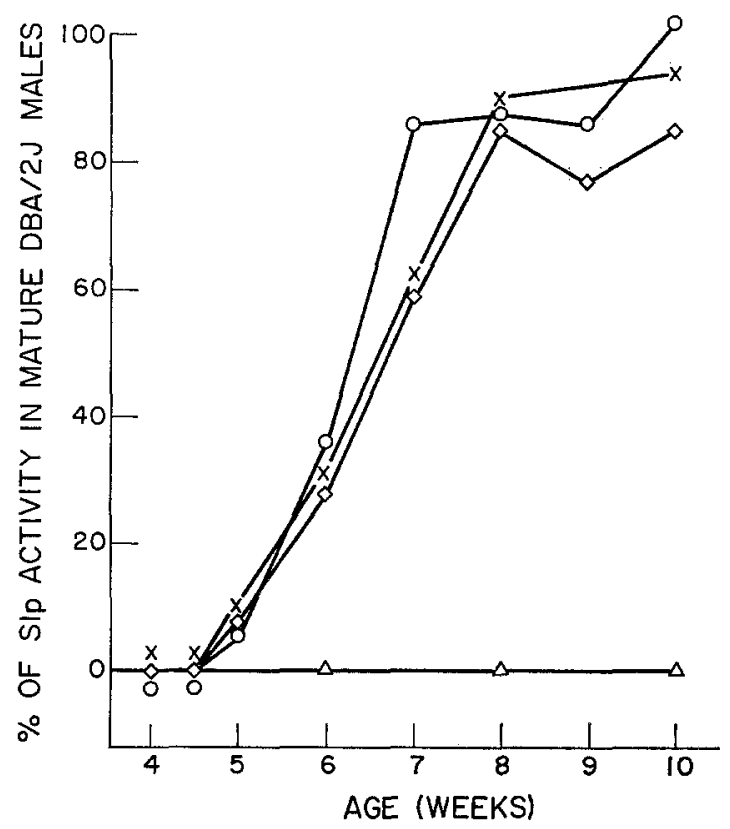

Fig. 2. The effect of testosterone propionate on immature DBA/2J females: $-\mathrm{O}-$ ovariectomized + testosterone beginning at $3 \frac{1}{2}$ weeks of age (eight animals); $-\diamond-$ females + testosterone beginning at $3 \frac{1}{2}$ weeks of age (eight animals); $-\Delta-$ ovariectomized + sesame oil beginning at $3 \frac{1}{2}$ weeks (four animals); $-\times$-females + testosterone beginning at 1 week of age (four animals).

The effect of treating mature, 12-week-old DBA/2J females with testosterone propionate is shown in Fig. 4. As was the case with immature females, the treatment results in the development of full adult levels of Slp. When the hormone treatment was suspended after 39 days, the level of Slp antigen declined and was not detectable 80 days after suspension of treatment. Control females, not treated with hormone, did not develop Slp antigen. Females from the genotypically negative strain C57BL/10JSf failed to develop Slp antigen when treated with hormone.

To determine whether increasing the dose of testosterone propionate would increase the rate of SIp appearance or the final level of the antigen, a group of adult females was treated (starting at 12 weeks of age) with a dose of hormone eightfold greater than that used in the previous experiments with adult females. The results of this experiment, illustrated in Fig. 4, clearly showed that the $8 \times$ dose did not increase the rate of Slp appearance or the final level of the antigen. On the contrary, the final level of antigen may have actually been reduced. Four females treated at the same time with the normal $(1 \times)$ dose of hormone responded identically to the $1 \times$ dose group in Fig. 4.

The quantitative level of Ss protein was followed in the experiments on immature 


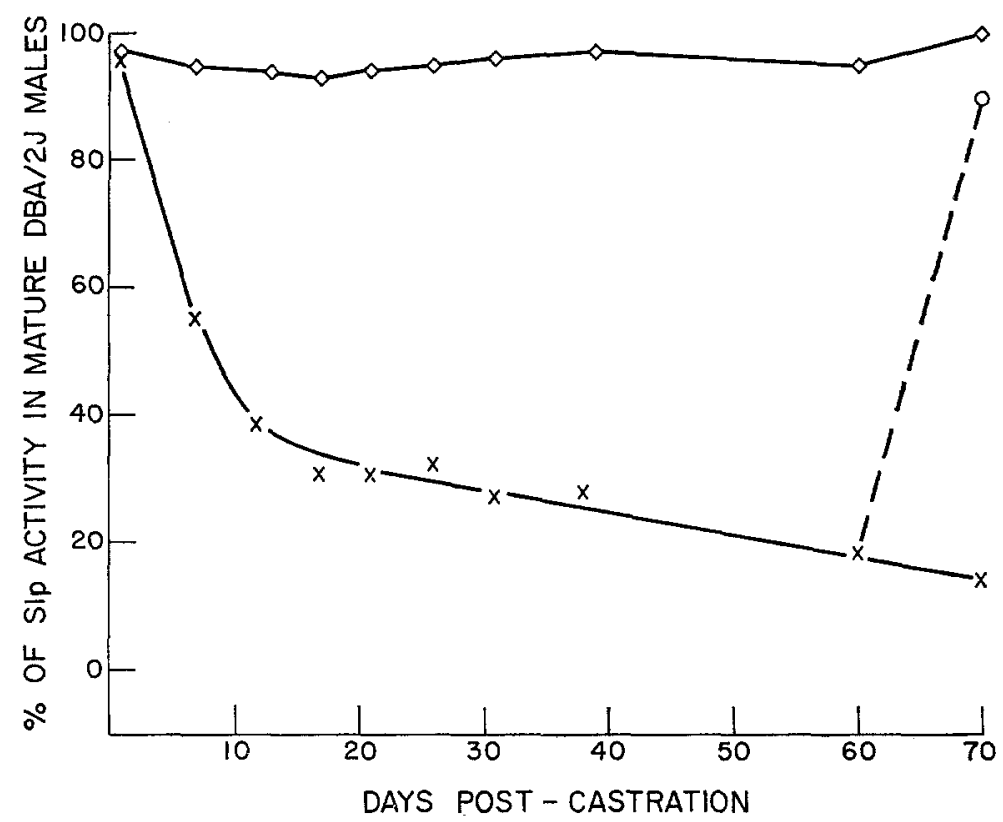

Fig. 3. The effect of castration on mature DBA/2J males (12 weeks of age): $-\diamond-$ sham control males (four animals); $-\times$-castrated at 12 weeks of age (nine animals); - - --- males treated with testosterone 60 days after castration (two animals).

DBA/2J animals (Figs. 1 and 2) to determine whether the development of Slp was correlated with any quantitative changes in Ss protein levels. This is of particular interest, because the Slp antigenic sites are believed to reflect structural differences in the Ss protein of different strains (Passmore and Shreffler, 1970). The quantitative level of Ss was approximately the same at 5 weeks of age in all groups of animals. At this age, the level was $50-60 \%$ of the adult DBA $/ 2 \mathrm{~J}$ male standard. At 10 weeks of age, Ss levels had increased to $80-100 \%$ in DBA/2J males, castrated DBA/2J males given hormone replacement, and DBA/2J females treated with testosterone. Ss levels remained unchanged at $50-60 \%$ in castrated DBA/2J males and normal females. Although the three groups showing increased Ss levels all developed Slp antigen, while the two groups showing no increase did not develop Slp antigen, similar quantitations of an Slp-negative strain, C57BL/10JSf, showed the same pattern of increase in Ss levels in normal males and treated females, with no increase in normal females. None of these C57BL/10JSf animals developed Slp antigen. Therefore, the increase in Ss levels is correlated only with the male hormonal environment and appears to occur in both Slp-positive and Slp-negative strains.

A number of experiments were performed to determine whether the hormone acts directly on the DBA/2J female serum to modify the Ss protein so that Slp activity is expressed, or whether the hormone triggers some intermediate step which allows for the appearance of Slp, e.g., the induction of an enzyme which in turn modifies the Ss 


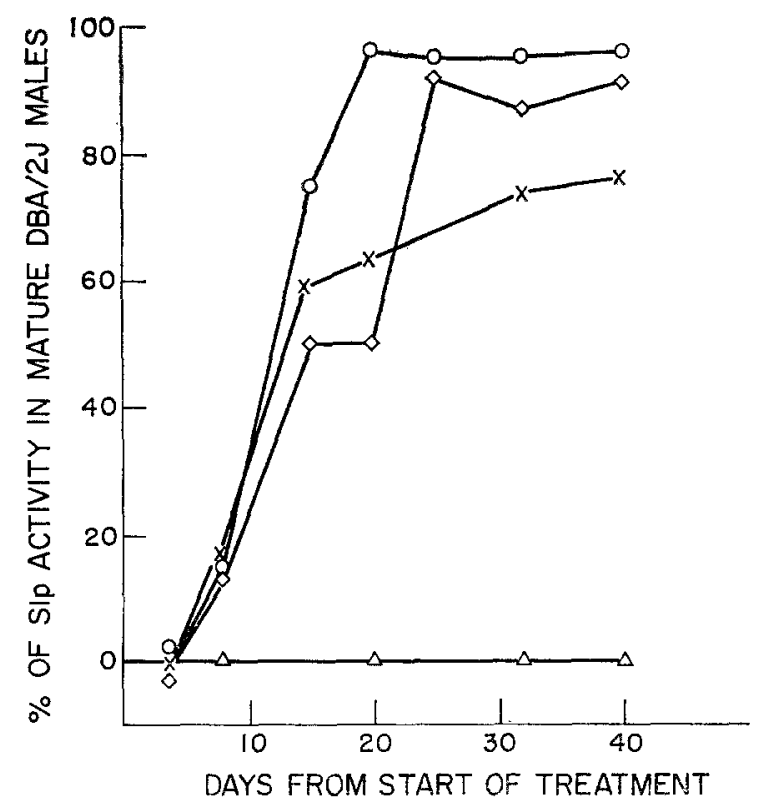

Fig. 4. The effect of testosterone propionate on mature DBA/2J females (12 weeks of age) :- - - ovariectomized + testosterone treatment (nine animals); $-\diamond-$ females + testosterone (six animals); $-\Delta-$ - ovariectomized + sesame oil (five animals); $-\times-$ females + eightfold increased dose of testosterone (four animals).

protein. When DBA/2J female serum was mixed in vitro with testosterone propionate or with male serum from the Slp-negative strain C57BL/10JSf, no SIp appeared after 24 and $48 \mathrm{hr}$ of incubation at either 37 or $25 \mathrm{C}$. Therefore, no direct modification of protein by the hormone could be demonstrated.

When $0.4 \mathrm{ml}$ of DBA/2J male whole serum was injected intraperitoneally into $\mathrm{DBA} / 2 \mathrm{~J}$ females or into C57BL/10JSf males daily for 8 days, Slp could be detected in the serum of the recipient animals for as long as $24 \mathrm{hr}$ after the last injection. This would indicate that the Slp antigen is absorbed from the peritoneal cavity into the circulation of the recipient animals. Presumably, if testosterone acted directly on the Ss protein from Slp-positive strains to produce Slp antigen, the injection of female serum from an Slp-positive strain into an Slp-negative male would provide the proper environment for modification of the $\mathrm{Ss}$, and therefore for the appearance of $\mathrm{Slp}$ antigen. However, when DBA/2J female whole serum was injected into C57BL/10JSf males using the same 8-day injection schedule, no Slp antigen could be detected $24 \mathrm{hr}$ after the last injection.

The original anti-Slp serum was produced by immunizing RF/J males with Ss protein from $\mathrm{DBA} / 2 \mathrm{~J}$ male serum. The results of a series of alloimmunizations involving either female donors or recipients are presented in Table I. Females from the Slp-negative strain $\mathrm{C} 3 \mathrm{H} / \mathrm{JSF}$ were capable of producing anti-Slp when immunized with 
Table I. Alloimmunizations Involving Female Recipients or Donors

\begin{tabular}{|c|c|c|c|}
\hline \multicolumn{2}{|c|}{ Strain combination } & \multirow{2}{*}{$\begin{array}{l}\text { Number } \\
\text { immunized }\end{array}$} & \multirow{2}{*}{$\begin{array}{c}\text { Number } \\
\text { producing anti-Slp }\end{array}$} \\
\hline Recipient & Donor & & \\
\hline $\mathrm{C} 3 \mathrm{H} / \mathrm{JSf} q$ & $\mathrm{~A} / \mathrm{J} \delta$ & 2 & 2 \\
\hline $\mathrm{C} 3 \mathrm{H} / \mathrm{JSf}$ ㅇ & $\mathrm{DBA} / 2 \mathrm{~J} \sigma$ & 2 & 2 \\
\hline $\mathbf{R F} / \mathbf{J}$ Q & $\mathrm{DBA} / 2 \mathrm{~J} q$ & 3 & 0 \\
\hline $\mathrm{RF} / \mathbf{J} \sigma$ & $\mathrm{DBA} / 2 \mathrm{~J} ?$ & 3 & 0 \\
\hline $\mathrm{A} / \mathrm{Sn}$ Q & $\mathrm{A} / \mathrm{Sn} \mathrm{o}^{x}$ & 3 & 0 \\
\hline
\end{tabular}

partially purified A/Sn or DBA/2J male serum. However, the immunization of $\mathrm{A} / \mathrm{Sn}$ females with a serum preparation from $\mathrm{A} / \mathrm{Sn}$ males produced no antibody, despite the fact that the donor was phenotypically positive and the recipient was phenotypically negative. This result would suggest that females from positive strains are tolerant to Slp antigen. It is, of course, possible that these females have very low levels of Slp antigen or that antigen is present but masked in some way. Partially purified Ss protein from DBA/2J females (phenotypically negative but genotypically positive) did not produce anti-Slp in animals of the Slp-negative strain $\mathrm{RF} / \mathrm{J}$. If, in fact, DBA/2J females have low levels of Slp or masked Slp, it is not of sufficient quantity to elicit an immune response in these $\mathrm{RF} / \mathrm{J}$ animals.

\section{DISCUSSION}

The castration and hormone treatment experiments with both immature and adult animals reveal that both the proper genotype and the presence of male hormone are required for the appearance of Slp antigen. Thus, the development of the SIp antigen clearly is a result of both genetic and hormonal factors. The disappearance of Slp antigen from the sera of castrated males, and from the sera of treated females after suspension of treatment, demonstrates that hormone is required for the maintenance of the antigen as well as for the triggering of its development.

Although it is evident that male hormone plays a primary role in the control of the phenotypic expression of the Slp variant, the precise mechanism for this control is unclear. Some preliminary clues to this mechanism can be discerned from the experimental results. The discussion which follows will be based on the assumption that the Slp variant reflects structural differences in the Ss protein among inbred strains of mice (Passmore and Shreffler, 1970).

Since the genetic studies show the Slp trait to be autosomally inherited, females from genotypically Slp-positive strains should have the genetic information necessary to code for the variant Ss protein structure. Therefore, in view of the fact that DBA/2J females can produce Slp antigen after treatment with testosterone, the absence of Slp antigen from the serum of normal untreated females must result from absence of the male hormone environment, not from inability to produce the variant protein. 
Since the time and rate of appearance of Slp antigen are the same in DBA/2J animals, whether hormone treatment is started at 1 week or $3 \frac{1}{2}$ weeks of age, it seems likely that a differentiation step independent of the presence of hormone must occur during postnatal development before hormone stimulation can be effective. Assuming that this hormone-independent differentiation is complete in mature $\mathrm{DBA} / 2 \mathrm{~J}$ females, the minimum lag time necessary to reach detectable levels of Slp antigen is the period of approximately 1 week from the start of treatment until appearance of Slp antigen in the serum of these adult females. Treated immature females develop the antigen at 5 weeks of age. If the lag time is approximately 1 week, then the hormone-independent differentiation step must be complete no earlier than 4 weeks of age and treatment before this time is ineffective.

It would appear possible, a priori, that the Ss protein from Slp-positive strains could have a basic primary structural difference which makes it susceptible to direct modification by male hormone. However, the failure of Slp to appear after direct exposure, either in vivo or in vitro, of DBA/2J female serum to hormone and the 6-8 day lag period between administration of hormone and appearance of Slp antigen in adult females argue against modification of the Ss protein either by hormone or by a circulating intermediate, such as an enzyme induced by the hormone.

Although a great deal of additional work will be necessary to elucidate the precise mechanism of hormonal control of the Slp antigen, the data presented in this report do reveal several important facts about the phenomenon: (1) Male hormone is required for the development and maintenance of Slp antigen in genotypically positive animals. (2) Females of Slp-positive strains have the genetic information necessary to code for and produce Slp antigen. (3) There is a differentiation step independent of male hormone which must be completed before Slp antigen can develop. (4) Male hormone probably does not act directly on Ss protein in the serum to modify it or to expose masked Slp antigen sites.

\section{REFERENCES}

Passmore, H. C. (1969). The influence of male hormone on a sex-limited serum protein variant in the house mouse. Genetics 61: s45.

Passmore, H. C., and Shreffler, D. C. (1970). A sex-limited serum protein variant in the mouse: Inheritance and association with the $H-2$ region. Biochem. Genet. 4: 351 .

Preer, J. R. (1956). A quantitative study of a technique of double diffusion in agar. J. Immunol. $77: 52$.

Shreffler, D. C. (1967). Genetic control of cellular antigens. In Crow, J. F., and Neel, J. V. (eds.), Proceedings of the Third International Congress on Human Genetics, Johns Hopkins Press, Baltimore, p. 217.

Shreffler, D. C., and Owen, R. D. (1963). A serological detected variant in mouse serum: Inheritance and association with the histocompatibility-2 locus. Genetics 48: 9.

Shreffler, D. C., and Steinberg, A. G. (1967). Further studies on the Xavante Indians. IV. serum protein groups and the $\mathrm{SC}_{1}$ trait of saliva in the Simoes Lopes and Sao-Marcos Xavantes. $A \mathrm{~m}$. J. Human Genet. 19: 514. 